\title{
Does transcranial direct current stimulation improve functional locomotion in people with Parkinson's disease? A systematic review and meta-analysis
}

\author{
Hyo Keun Lee ${ }^{1,2}$, Se Ji Ahn', Yang Mi Shin ${ }^{1}$, Nyeonju Kang ${ }^{1,3^{*}}$ (D) and James H. Cauraugh ${ }^{4}$
}

\begin{abstract}
Purpose: The purpose of this meta-analysis was to investigate the treatment effects of transcranial direct current stimulation (tDCS) on functional locomotion in people with Parkinson's disease (PD).

Methods: A systematic literature search identified 18 qualified studies that used tDCS protocols as functional locomotion rehabilitation interventions for people with PD. All included studies used either a randomized control trial or crossover designs with a sham control group. Meta-analysis quantified both (a) short-term treatment effects: change in functional locomotion between baseline and immediate posttests on 18 comparisons and (b) long-term treatment effects: change in functional locomotion between baseline and delayed retention tests on six comparisons. Moreover, we performed moderator variable analyses for comparing effect sizes between tDCS targeting multiple brain regions and tDCS targeting a single brain region.

Results: Random effects model meta-analyses revealed a significant short-term treatment effect (effect size $=0.359$; $P=0.001$ ), whereas no significant long-term treatment effects were identified (effect size $=0.164 ; P=0.314$ ). In addition, tDCS protocols that targeted multiple brain regions showed relatively more positive effects on functional locomotion than protocols that targeted a single brain region.

Conclusions: These meta-analytic findings indicate that tDCS protocols may show immediate positive effects on functional locomotion in people with PD. However, given the relatively low effect size, exploring more appropriate tDCS protocols (i.e., targeting multiple motor and prefrontal regions and medication condition) should be a focus in future studies.
\end{abstract}

Keywords: Parkinson's disease, Functional locomotion, Transcranial direct current stimulation, Meta-analysis

\section{Background}

Parkinson's disease (PD) is a neurodegenerative disease attributed to progressive degeneration of dopamineproducing neurons within the basal ganglia mainly affecting the motor cortex [1]. The cardinal symptoms of PD are manifested as motor related features including bradykinesia, rigidity, resting tremor, postural instability, and gait disturbance [2]. Applying medications such as levodopa and carbidopa, chemical supplements for increasing

\footnotetext{
* Correspondence: nyunju@inu.ac.kr

'Division of Sport Science, Neuromechanical Rehabilitation Research Laboratory, Incheon National University, 119 Academy-ro, Yeonsu-gu, Incheon, South Korea

${ }^{3}$ Sport Science Institute, Incheon National University, Incheon, South Korea Full list of author information is available at the end of the article
}

dopamine, may be an efficient clinical option for improving rigidity and slowness of movement of people with PD [3]. However, the pharmacological treatments are less effective as the disease progresses $[4,5]$. Deep brain stimulation (DBS), a surgical approach, has been introduced and complementally used particularly for people with PD with moderate to severe disease severity [6]. Despite the evidence of treatment effects on motor symptoms of PD after DBS $[7,8]$, this neurosurgical option is cautiously used for PD treatment because of high cost and potential surgical risk $[9,10]$. Therefore, exploring therapeutic alternatives and rehabilitation interventions as a complementary treatment is still required.

(C) The Author(s). 2019 Open Access This article is distributed under the terms of the Creative Commons Attribution 4.0 International License (http://creativecommons.org/licenses/by/4.0/), which permits unrestricted use, distribution, and 
Recently, neurorehabilitation researchers have increased their attention on the utility of non-invasive brain stimulations as therapeutic alternatives for treating motor symptoms of PD [11]. In particular, transcranial direct current stimulation (tDCS) which is one of the non-invasive brain stimulation (NIBS) techniques has been investigated for PD motor recovery [12]. Despite insufficient findings regarding neurophysiological mechanisms underlying tDCS, this intervention may be an attractive rehabilitation option because of its practical advantages of economic efficiency, portability, and accessibility. Basically, tDCS provides both anodal and cathodal stimulations by delivering weak direct currents (e.g., intensity $=1-2 \mathrm{~mA}$ ) to the scalp via surface electrodes. Based on potential mechanisms of tDCS that anodal tDCS increases cortical excitability and cathodal tDCS decreases cortical excitability [13], tDCS may reorganize neural activation patterns and facilitate neural plasticity in specific targeted regions of the brain [14, 15]. Perhaps, tDCS can potentially modulate functional connectivity among the cortico-striatal and thalamocortical circuits of brain [16]. These neuronal alterations by tDCS can provide functional advantages for PD motor rehabilitation. Moreover, Quartarone et al. [17] reported that the modulation of neuronal excitability may last beyond the stimulating period supporting the suggestion that $\mathrm{tDCS}$ may be effective for improving motor symptoms in people with PD.

Several studies using animal models showed tDCS findings modulating dopaminergic pathways [18, 19]. Specifically, anodal tDCS could activate dopaminergic neurons and promote dopamine levels of striatum in a monkey and rats with PD $[18,20]$. These findings support the potential efficacy of tDCS in motor rehabilitation of people with PD [21]. For a human model, tDCS protocols primarily targeted motor and prefrontal cortices (e.g., primary motor cortex: M1 and dorsolateral prefrontal cortex: DLPFC) because brain activation patterns in these brain regions are highly involved in successful locomotion performance in people with PD [22-25]. Fregni et al. [22] suggested that greater M1 activation after anodal tDCS was related to improvements in motor function of PD. Further, people with PD revealed higher DLPFC activation during normal walking because they presumably compensated for deficits in gait automaticity by increasing cognitive control (e.g. executive control). Thus, more DLPFC activation by anodal tDCS may be necessary when people with PD completed more complex locomotion tasks [23-25]. Moreover, some repetitive transcranial magnetic stimulation (rTMS) studies reported release of dopamine in the caudate and putamen in healthy individuals $[26,27]$ and people with PD [28] when the stimulation triggered motor and prefrontal cortical regions. Presumably, applying tDCS may cause similar dopamine release contributing to acute motor improvements as well.

Two previous meta-analysis studies reported the overall positive effects of various NIBS techniques including rTMS, tDCS, and transcranial alternating current stimulation (tACS) on various motor symptoms in $[29,30]$. However, the prior meta-analytic findings regarding motor improvement evidence of people with PD were estimated by heterogeneous outcome measures (e.g., tremor, rigidity, gait, and bradykinesia) and different NIBS protocols. Importantly, a recent systematic review study by Broeder et al. [31] suggested potential treatment effects of tDCS protocols on gait performance in people with $\mathrm{PD}$. Locomotion impairment is one recognizable motor symptom compromising independence and quality of life in people with PD, and effectively represents an individual's progression of disease severity [8]. Although a recent meta-analysis by Goodwill et al. [29] reported significant positive effects of tDCS and tACS on gait functions, these meta-analytic findings were still limited to small sample sizes (i.e., two studies). Thus, the current systematic review and meta-analysis investigated the treatment effects of tDCS on functional locomotion in people with PD. Moreover, given that multiple cerebral regions related to motor and cognitive functions may influence functional locomotion, we addressed an additional question: Do tDCS protocols targeting multiple brain regions and a single brain region reveal similar treatment effects on functional locomotion?

\section{Methods}

\section{Literature search and study selection}

The current meta-analyses were conducted consistent with the suggestions by the Preferred Reporting Items for Systematic Reviews and Meta-Analyses (PRISMA) statement consisting of a checklist and a flow diagram [32]. Especially, this study reported all PRISMA checklist items (Additional file 1), and failed to register the systematic review protocol because we already completed data extraction and analyses. We conducted a computerized literature search within July 2018-May 2019 using PubMed, Web of Science, and Cochrane Databased of Systematic Reviews. All types of publications were considered regardless of publication date. Search terms were: (a) Parkinson or Parkinson's disease or PD, (b) transcranial direct current stimulation or transcranial electrical stimulation or tDCS, and (c) gait or walk or walking or locomotion or locomotor task. The inclusion criteria of this meta-analysis included: (a) reporting quantitative data related to functional locomotion, (b) including between-group comparisons (i.e., active tDCS versus sham tDCS), and (c) using either a crossover design or randomized control trial design. 
Extraction of functional locomotion outcome measures We analyzed functional locomotion by investigating individual's temporal components (i.e., gait speed and time to complete specific task) in various functional locomotion tests. Moreover, we estimated the treatment effects of tDCS on functional locomotion in PD for two different perspectives: (a) short-term effects indicating changes in functional locomotion (i.e., retention time between baseline and immediate posttest $\leq 24 \mathrm{~h}$ after final tDCS intervention) and (b) long-term effects denoting changes in functional locomotion (i.e., retention time between baseline and follow-up tests $\geq 4$ weeks after final tDCS intervention).

\section{Methodological quality assessments}

Two authors (SA and YS) independently assessed the methodological quality for the qualified studies using the Physiotherapy Evidence Database (PEDro) rating scale [33], and further estimated the risk of bias of all studies using the Cochrane risk of bias assessment [34]. The PEDro scale estimated study quality using a checklist of 10 items scored yes-or-no related to group allocation, blinding, attrition, statistical analyses, and data variability. Using Review Manager 5.3 software (Copenhagen: The Nordic Cochrane Centre, The Cochrane Collaboration, 2014), we additionally performed the Cochrane risk of bias assessment estimating (a) random sequence allocation, (b) allocation concealment, (c) blinding of participants and personnel, (d) blinding of outcome assessment, (e) incomplete outcome data, (f) selective reporting, and $(\mathrm{g})$ other sources of bias. Discrepancies in quality assessment scores between the two authors activated a third assessor (NK) who separately graded and confirmed the final scores.

\section{Meta-analytic techniques}

We conducted the meta-analyses using the Comprehensive Meta-Analysis software (ver. 3.0. Englewood, NJ, USA). For quantifying individual effect sizes, we calculated standardized mean difference $(S M D)$ and $95 \%$ confidence intervals (CIs) for each comparison. SMDs and CIs for 18 short-term treatment comparisons and six long-term treatment comparisons were estimated. The current meta-analyses used a random effects model because we posited that individual effect sizes are different and no common effect size across comparisons exists because of different participant populations, treatment protocols, and statistical designs [35]. In addition, we conducted a moderator variable analysis quantifying overall effect sizes between two sub-groups: (a) tDCS targeting multiple brain regions versus (b) tDCS targeting a single brain region. However, we examined the moderator variable analysis for the short-term treatment effects of tDCS only because of insufficient number of comparisons for the long-term treatment effects of tDCS.
To quantify variability of individual effect sizes across qualified studies, we performed three heterogeneity tests: (a) Cochran's $Q$, (b) $T^{2}$ (estimate of tau-squared) and (C) Higgins and Green's $I^{2}$. Cochran's $Q$ test provides $\mathrm{Q}$ statistics with $P$-value at alpha level equal to 0.05 . $P$-value less than 0.05 indicated a significant heterogeneity across studies [35]. The level of $T^{2}$ is an estimate of variance of the observed effects with weights assigned in a random effects model so that $T^{2}$ greater than 1.0 denotes a significant level of variability across studies [36, 37]. Finally, $I^{2}$ is the percentage of the heterogeneity, and further $I^{2}$ higher than $50 \%$ indicates substantial between-studies heterogeneity [38].

The publication bias indicating the risk of bias across studies was estimated with three techniques: (a) funnel plot, (b) Egger's regression test, and (c) Begg and Mazumdar rank correlation test. One conventional approach is to visually estimate publication bias level via the funnel plot displaying $S M D$ vs. standard error for each comparison. Before and after applying trim and fill technique [39], we compared two overall effect sizes between the original plot and the revised plot with potential imputed values. Lower changes in the overall effect sizes with minimum number of imputed values potentially indicates a minimal level of publication bias across studies. Egger's regression and Begg and Mazumdar rank correlation tests are quantitative approaches to measuring the level of publication bias. Egger's regression test provides the asymmetry of funnel plot by calculating the intercept from regression of standard normal deviates versus precision so that an intercept with $P$-value greater than 0.05 indicates minimal publication bias [40]. Similarly, Begg and Mazumdar rank correlation test shows the correlation between the ranks of effect sizes and the ranks of their variances. Thus, the rank correlation (Kendall's Tau) with $P$-values greater than 0.05 denotes minimal risk of bias across studies [41].

\section{Results \\ Study identification}

The PRISMA flow diagram in Fig. 1 shows the specific steps for the study identification procedures. Initially, our literature search identified 147 potential studies, and excluded 64 duplicated articles. After reviewing the abstract and text for each study based on our inclusion and exclusion criteria by three authors (NK, SA, and YS), we excluded 65 studies because of review articles, not-related disease, no functional locomotion results reported (e.g., protocol study), and not applying tDCS. Finally, 18 total studies met our inclusion criteria were qualified for the meta-analysis [42-59].

\section{Participant characteristics}

The 18 qualified studies included 325 people with PD (range of mean age $=56.7-72.3$ years). A range of mean 


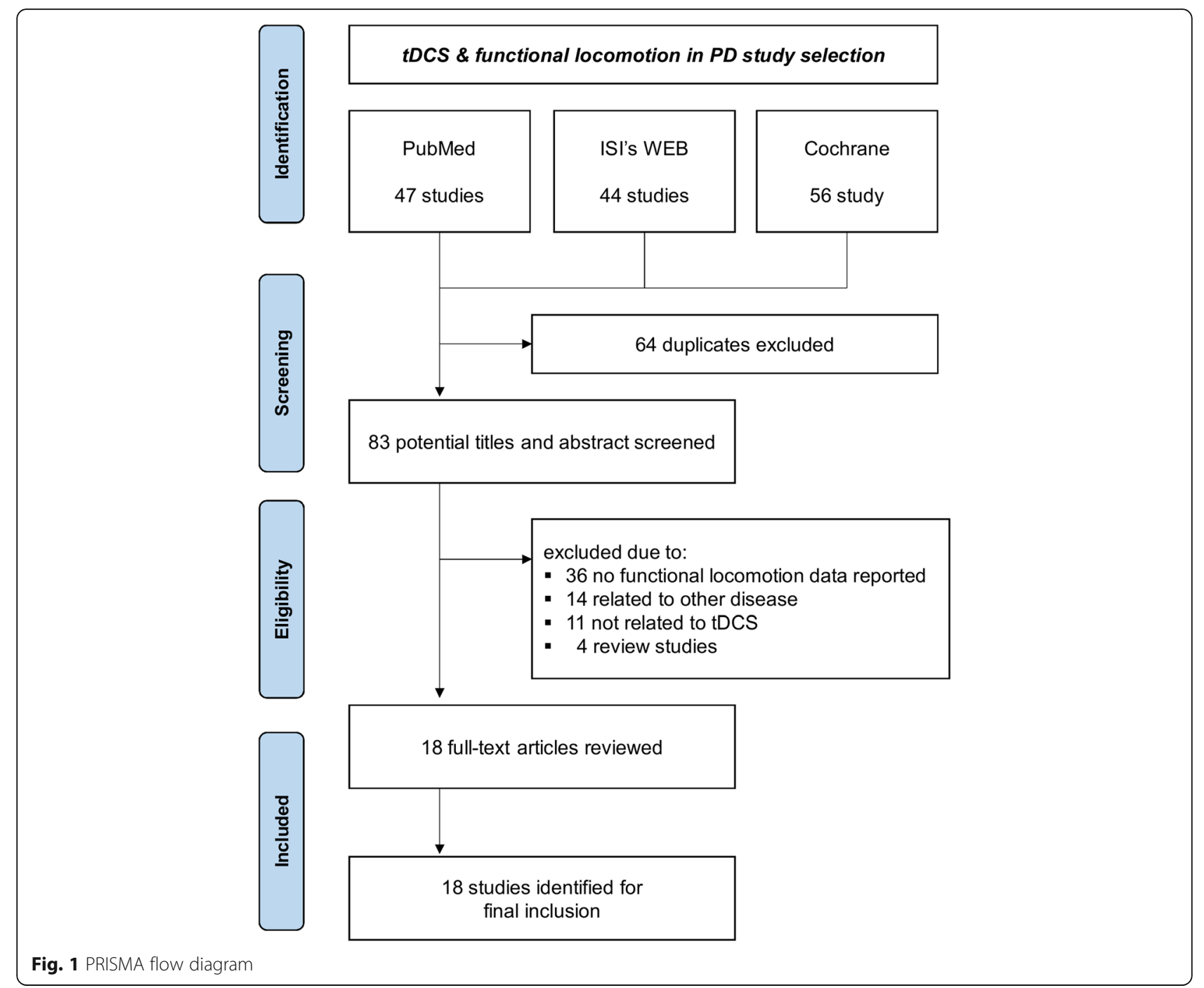

period beyond PD diagnosis was 4.6-16.8 years. A range of mean motor examination scores in The Unified Parkinson's Disease Rating Scale at baseline equaled 11.247.7 (higher scores indicate worse motor functions). Fourteen out of 18 total studies reported medication status of participants that all people with PD were on medication, and the remaining four studies did not mention medication status. Specific details regarding participant characteristics are shown in Table 1.

\section{tDCS intervention protocols}

Table2 shows specific tDCS parameters for the qualified studies. All 18 included studies used active tDCS (i.e., anodal tDCS: 16 studies and anodal \& cathodal tDCS: two studies) and sham stimulation. Targeted brain regions of active tDCS for the qualified studies included prefrontal cortex (PFC), dorsal lateral prefrontal cortex (DLPFC), premotor cortex (PMC), supplementary motor area (SMA), primary motor cortex (M1; C3 or C4 in the
International $10-20$ system), and leg areas of $\mathrm{M} 1(\mathrm{Cz}$ in the International 10-20 system). Six studies stimulated multiple brain regions (e.g., one bilateral PFC and M1; one bilateral PFC, PMC, and M1; one bilateral PMC and M1; two bilateral DLPFC; one M1 and left-DLPFC) whereas 12 studies used a single targeted brain region. Six out of 12 studies that used a single targeted brain region applied anodal tDCS on the central leg areas of M1, and the remaining six studies targeted either M1 or DLPFC of one side of hemisphere (one leg area of M1 of affected hemisphere; three M1 of left hemisphere; one left DLPFC; one right DLPFC). Finally, seven studies used a single session of tDCS protocols and 11 studies applied multiple sessions of tDCS protocols.

\section{Functional locomotion outcome measures}

Eighteen qualified studies reported one of following outcome measures: (a) gait speed: eight studies and (b) the time that a person takes to complete certain locomotion 
Table 1 Participant characteristics

\begin{tabular}{|c|c|c|c|c|c|c|c|c|}
\hline Study & Total N & Age (yrs) & Gender & $\begin{array}{l}\text { PD Duration } \\
\text { (yrs) }\end{array}$ & $\begin{array}{l}\text { UPDRS Part III } \\
\text { at Baseline }\end{array}$ & Medication & $\begin{array}{l}\text { DBS } \\
\text { Treatment }\end{array}$ & FOG Test \\
\hline Alizad [42] & 8 & NA & Total: 3F, $5 \mathrm{M}$ & NA & NA & NA & NO & NA \\
\hline Benninger [43] & 25 & Total: $63.9 \pm 8.7$ & $\begin{array}{l}\text { Active: } 4 \mathrm{~F}, 9 \mathrm{M} \\
\text { Sham: } 5 \mathrm{~F}, 7 \mathrm{M}\end{array}$ & $\begin{array}{l}\text { Active: } 10.6 \pm 7.1 \\
\text { Sham: } 9.1 \pm 3.3\end{array}$ & $\begin{array}{l}\text { Active: } 22.2 \pm 8.7 \\
\text { Sham: } 17.5 \pm 8.0\end{array}$ & On & NO & $\begin{array}{l}\text { Patients with } \\
\text { severe freezing or } \\
\text { unable to walk } 10 \\
\text { m were excluded }\end{array}$ \\
\hline Capacci [44] & 7 & Total: $60.9 \pm 9$ & Total: 4F, $3 \mathrm{M}$ & Total: $16.8 \pm 4.0$ & NA & NA & NO & NA \\
\hline Costa-Ribeiro [45] & 22 & $\begin{array}{l}\text { Active: } 61.1 \pm 9.1 \\
\text { Sham: } 62.0 \pm 16.7\end{array}$ & $\begin{array}{l}\text { Active: } 3 \mathrm{~F}, 8 \mathrm{M} \\
\text { Sham: } 4 \mathrm{~F}, 7 \mathrm{M}\end{array}$ & $\begin{array}{l}\text { Active: } 6.1 \pm 3.8 \\
\text { Sham: } 6.3 \pm 3.7\end{array}$ & $\begin{array}{l}\text { Active: } 19.0 \\
\text { Sham: } 19.1\end{array}$ & On & NO & $\begin{array}{l}\text { FOG-Q(> } 15 \\
\text { points) were } \\
\text { excluded }\end{array}$ \\
\hline Costa-Ribeiro [46] & 22 & $\begin{array}{l}\text { Active: } 61.1 \pm 9.1 \\
\text { Sham: } 62.0 \pm 16.7\end{array}$ & $\begin{array}{l}\text { Active: } 3 \mathrm{~F}, 8 \mathrm{M} \\
\text { Sham: } 4 \mathrm{~F}, 7 \mathrm{M}\end{array}$ & $\begin{array}{l}\text { Active: } 6.1 \pm 3.8 \\
\text { Sham: } 6.3 \pm 3.7\end{array}$ & $\begin{array}{l}\text { Active: } 19.0 \pm 4.9 \\
\text { Sham: } 17.6 \pm 5.1\end{array}$ & On & NO & $\begin{array}{l}\text { Patients were } \\
\text { excluded when } \\
\text { they presented } \\
\text { severe freezing } \\
\text { according the } \\
\text { FOG-Q }\end{array}$ \\
\hline Criminger [47] & 16 & Total: $68.1 \pm 9.8$ & Total: 4F, $12 \mathrm{M}$ & Total: $8.7 \pm 9.8$ & Total: $23.4 \pm 9.7$ & On & NO & NA \\
\hline da Silva [48] & 17 & $\begin{array}{l}\text { Active: } 66.0 \pm 5.0 \\
\text { Sham: } 66.0 \pm 10.0\end{array}$ & $\begin{array}{l}\text { Active: } 4 \mathrm{~F}, 4 \mathrm{M} \\
\text { Sham: } 3 \mathrm{~F}, 6 \mathrm{M}\end{array}$ & $\begin{array}{l}\text { Active: } 6.0 \pm 6.0 \\
\text { Sham: } 5.0 \pm 1.0\end{array}$ & NA & NA & NO & NA \\
\hline Dagan [49] & 20 & NA & NA & NA & NA & On & NO & $\begin{array}{l}\text { FOG-Q: } 20.5 \pm 4.9 \\
\text { FOG-provoking test } \\
\text { scores: } 14.2 \pm 8.00\end{array}$ \\
\hline Fernández-Lago [50] & 18 & Total: $56.7 \pm 11.6$ & Total: 7F, $11 \mathrm{M}$ & Total: $6.2 \pm 3.7$ & Total: $21.17 \pm 11.3$ & On & NO & NA \\
\hline Kaski [51] & 16 & NA & NA & NA & NA & On & NO & $\begin{array}{l}\text { Patients with } \\
\text { severe freezing } \\
\text { were excluded }\end{array}$ \\
\hline Lattari [52] & 17 & Total: $67.2 \pm 10.0$ & Total: 4F, $13 \mathrm{M}$ & Total: $7.1 \pm 2.7$ & Total: $18.0 \pm 99.0$ & On & NO & NA \\
\hline Mak [53] & 18 & NA & NA & NA & NA & NA & NO & NA \\
\hline Manenti [54] & 10 & Total: $67.1 \pm 7.2$ & Total: 4F, $6 \mathrm{M}$ & Total: $8.1 \pm 3.5$ & Total: $13.3 \pm 5.7$ & On & NO & NA \\
\hline Schabrun [55] & 16 & $\begin{array}{l}\text { Active: } 72.0 \pm 4.9 \\
\text { Sham: } 63.0 \pm 11.0\end{array}$ & $\begin{array}{l}\text { Active: } 8 \mathrm{M} \\
\text { Sham: } 6 \mathrm{~F}, 2 \mathrm{M}\end{array}$ & $\begin{array}{l}\text { Active: } 6.9 \pm 4.4 \\
\text { Sham: } 4.6 \pm 3.9\end{array}$ & $\begin{array}{l}\text { Active: } 47.7 \pm 7.5 \\
\text { Sham: } 37.7 \pm 9.8\end{array}$ & On & NO & NA \\
\hline Swank [56] & 10 & Total: $68.7 \pm 10.2$ & Total: 2F, $8 \mathrm{M}$ & Total: $7.9 \pm 7.1$ & Total: $37.0 \pm 12.9$ & On & NO & NA \\
\hline Valentino [57] & 10 & Total: $72.3 \pm 3.6$ & Total: 5F, $5 \mathrm{M}$ & Total: $11.0 \pm 4.9$ & Total: $32.0 \pm 10.3$ & On & NO & FOG-Q: $15.3 \pm 2.7$ \\
\hline Verheyden [58] & 20 & NA & NA & Total: $9.0 \pm 4.0$ & Total: $16.0 \pm 5.0$ & On & NO & NA \\
\hline Yotnuengnit [59] & 53 & $\begin{array}{l}\text { Active: } 68.2 \pm 9.8 \\
\text { Sham: } 62.7 \pm 8.8\end{array}$ & $\begin{array}{l}\text { Active: } 6 \mathrm{~F}, 11 \mathrm{M} \\
\text { Sham: } 6 \mathrm{~F}, 12 \mathrm{M}\end{array}$ & $\begin{array}{l}\text { Active: } 9.4 \pm 5.3 \\
\text { Sham: } 6.6 \pm 3.6\end{array}$ & $\begin{array}{l}\text { Active: } 11.9 \pm 4.7 \\
\text { Sham: } 11.2 \pm 4.0\end{array}$ & On & NO & NA \\
\hline
\end{tabular}

Abbreviations: Active Active tDCS protocols, DBS Deep brain stimulation, F Female, FOG Freezing of gait, FOG-Q Freezing of gait questionnaire, $M$ Male, NA Not applicable, PD Duration Time since PD diagnosis, UPDRS The Unified Parkinson's Disease Rating Scale. Note. Data for age and PD duration are mean \pm standard deviation

tasks: eight studies for Timed Up and Go test (TUG), one study for $10 \mathrm{~m}$ walking time, and one study for Stand Walk Sit test. All included studies reported shortterm effects of tDCS on functional locomotion, and six studies of 18 total studies reported long-term effects (mean $\pm \mathrm{SD}$ of retention time $=7.3 \pm 3.9$ weeks). For short-term effects of tDCS, seven studies reported functional locomotion difference between active and sham tDCS groups at posttest whereas 11 studies reported changes in functional locomotion between baseline and posttest after active tDCS as compared with sham stimulation. For long-term effects of tDCS, four studies reported functional locomotion difference between active and sham tDCS groups at posttest whereas two studies reported changes in functional locomotion between baseline and posttest after active tDCS as compared with sham stimulation.

Methodological quality assessments over included studies An average value of PEDro score was $7.7(S D=1.8)$, and this level indicates relatively good methodological quality across the included studies (Table 3). Moreover, we performed Cochrane's methodological quality assessment for estimating the risk of bias within each study. Figure 2 displays the risk of bias summary and graph indicating relatively low risk of bias for each study except for the selective bias. 
Table 2 tDCS protocols

\begin{tabular}{|c|c|c|c|c|c|c|}
\hline Study & Treatment & Session \# & Active tDCS & Stimulation Site & $\begin{array}{l}\text { Stimulation Parameters } \\
\text { (Intensity, Duration, Areas) }\end{array}$ & Follow-Up Test \\
\hline Alizad [42] & tDCS & 3 & A & M: Bi PMC \& M1 & $1 \mathrm{~mA}, 20 \mathrm{~min}, 40 \mathrm{~cm}^{2}$ & No \\
\hline Benninger [43] & tDCS & 8 & A & M: Bi PFC, PMC, \& M1 (separately) & $2 \mathrm{~mA}, 20 \mathrm{~min}, 24.5 \mathrm{~cm}^{2}$ & Yes (12wks) \\
\hline Capacci [44] & tDCS & 1 & A & M: Bi PFC (separately) & $2 \mathrm{~mA}, 20 \mathrm{~min}, \mathrm{NA}$ & No \\
\hline Costa-Ribeiro [45] & tDCS\&GT & 10 & A & $\begin{array}{l}\text { S: Central leg areas } \mathrm{M} 1 \\
(2 \mathrm{~cm} \text { anterior to the vertex })\end{array}$ & $2 \mathrm{~mA}, 13 \mathrm{~min}, \mathrm{NA}$ & Yes (4wks) \\
\hline Costa-Ribeiro [46] & tDCS\&GT & 10 & A & $\begin{array}{l}\text { S: Central leg areas } \mathrm{M} 1 \\
(2 \mathrm{~cm} \text { anterior to the vertex })\end{array}$ & $2 \mathrm{~mA}, 13 \mathrm{~min}, 35 \mathrm{~cm}^{2}$ & Yes (4wks) \\
\hline Criminger [47] & tDCS & 3 & $A \& C$ & M: Bi DLPFC (A-tDCS on LH \& C-tDCS on RH) & $2 \mathrm{~mA}, 20 \mathrm{~min}, 15 \mathrm{~cm}^{2}$ & No \\
\hline da Silva [48] & tDCS & 1 & A & S: Central leg areas M1 \& SMA & $2 \mathrm{~mA}, 15 \mathrm{~min}, 35 \mathrm{~cm}^{2}$ & No \\
\hline Dagan [49] & tDCS & 2 & A & M: M1 \& LH-DLPFC & $20 \mathrm{~min}, \mathrm{NA}, \mathrm{NA}$ & No \\
\hline Fernández-Lago [50] & tDCS\&TT & 1 & A & S: leg area $\mathrm{M} 1 \mathrm{of} \mathrm{AH}$ & $2 \mathrm{~mA}, 20 \mathrm{~min}, 3.5 \mathrm{~cm}^{2}$ & No \\
\hline Kaski [51] & tDCS\&PT & 1 & A & $\begin{array}{l}\text { S: Central leg areas M1 } \\
\text { (10-20\% anterior to the vertex) }\end{array}$ & $2 \mathrm{~mA}, 15 \mathrm{~min}, 40 \mathrm{~cm}^{2}$ & No \\
\hline Lattari [52] & tDCS & 1 & A & S: LH DLPFC & $2 \mathrm{~mA}, 20 \mathrm{~min}, 35 \mathrm{~cm}^{2}$ & No \\
\hline Mak [53] & tDCS & 5 & A & S: M1 & $\mathrm{NA}, 20 \mathrm{~min}, \mathrm{NA}$ & No \\
\hline Manenti [54] & tDCS & 2 & A & S: RH DLPFC & $2 \mathrm{~mA}, 7 \mathrm{~min}, 35 \mathrm{~cm}^{2}$ & No \\
\hline Schabrun [55] & tDCS\&GT & 9 & A & S: LH M1 & $2 \mathrm{~mA}, 20 \mathrm{~min}, 35 \mathrm{~cm}^{2}$ & Yes (12wks) \\
\hline Swank [56] & tDCS & 1 & $A \& C$ & M: Bi DLPFC (A-tDCS on LH \& C-tDCS on RH) & $2 \mathrm{~mA}, 20 \mathrm{~min}, \mathrm{NA}$ & No \\
\hline Valentino [57] & tDCS & 5 & A & S: Central leg areas M1 & $2 \mathrm{~mA}, 20 \mathrm{~min}, \mathrm{NA}$ & Yes (4wks) \\
\hline Verheyden [58] & tDCS & 1 & A & S: LH M1 & $1 \mathrm{~mA}, 15 \mathrm{~min}, \mathrm{NA}$ & No \\
\hline Yotnuengnit [59] & tDCS\&PT & 6 & A & S: Central leg areas M1 & $2 \mathrm{~mA}, 30 \mathrm{~min}, 35 \mathrm{~cm}^{2}$ & Yes (8wks) \\
\hline
\end{tabular}

Abbreviations: A Anodal tDCS, AH Affected hemisphere, Bi Bilateral, C Cathodal tDCS, DLPFC Dorsolateral prefrontal cortex, GT Gait training, $L H$ Left hemisphere, $M$ Multiple targeted brain regions, M1 Primary motor cortex, NA Not applicable, PFC Prefrontal cortex, PMC Premotor cortex, PT Physical training, RH Right hemisphere, $S$ Single targeted brain region, $\pi$ Treadmill training, wks Weeks (retention period)

\section{Meta-analytic results}

A random-effects model meta-analysis on the 18 comparisons from the qualified studies regarding the short-term treatment effects of $\mathrm{tDCS}$ revealed a significant overall effect size $(S M D=0.359 ; S E=0.105 ;$ Variance $=0.011 ; 95 \%$ $\mathrm{CI}=0.153-0.565 ; \mathrm{Z}=3.411 ; P=0.001)$. The current level of overall effect size indicates a relatively small positive effect [60]. The individual weighted effect sizes across 18 comparisons are shown in Fig. 3. These findings indicate that applying tDCS slightly improved functional locomotion in people with PD, and these results were short-term treatment effects that appeared at the immediate posttest.

For the short-term treatment effects of tDCS, heterogeneity test results were: (a) $Q$-statistics $=26.524$ and $P$-value $=$ 0.065 , (b) $T^{2}=0.067$, and (c) $I^{2}=35.907 \%$. These findings indicate a relatively low level of individual effect size variability across the 18 comparisons. Moreover, publication bias was minimal because both the original and revised funnel plots after the trim and fill technique shows relatively similar overall effect sizes with two imputed values (Fig. 4a). This finding was additionally confirmed by two quantitative publication bias tests: (a) Egger's regression test: intercept $\beta_{0}=$ 1.281 and $P$-value $=0.250$ and (b) Begg and Mazumdar rank correlation test: rank correlation $\mathrm{\tau}=0.163$ and $P$-value $=$
0.343. Taken together, these meta-analytic findings indicate that the positive short-term effects of tDCS on functional locomotion had minimal heterogeneity and risk of bias across the included PD studies.

Moreover, we performed two additional sensitivity analyses to determine whether overall effect sizes were different across three functional locomotion tasks (gait speed vs. TUG time vs. stand walk sit test) and two functional locomotion quantification approaches (difference between active and sham tDCS groups at posttest vs. changes between baseline and posttest after active tDCS as compared with sham stimulation). The first sensitivity analysis revealed two significant positive effects from multiple comparisons: (a) nine gait speed comparisons: $S M D=0.307 ; S E=0.151$; Variance $=0.023 ; 95 \% \mathrm{CI}=0.011-0.603 ; \mathrm{Z}=2.030 ; P=0.042$, (b) eight TUG time comparisons: $S M D=0.365 ; S E=0.164$; Variance $=0.027 ; 95 \% \mathrm{CI}=0.044-0.686 ; \mathrm{Z}=2.229 ; \quad P=$ 0.026 , and (c) one stand walk sit test comparison: $S M D=$ $0.715 ; S E=0.354$; Variance $=0.126 ; 95 \% \mathrm{CI}=0.021-1.410$; $\mathrm{Z}=2.019 ; P=0.044$. The second sensitivity analysis showed two significant positive effects: (a) 11 difference at posttest comparisons: $S M D=0.260 ; S E=0.119$; Variance $=0.014$; 95\% CI $=0.027-0.494 ; \mathrm{Z}=2.188 ; P=0.029$ and (b) seven changes from baseline to posttest comparisons: $S M D=$ 
Table 3 PEDro score for methodological quality assessment

\begin{tabular}{|c|c|c|c|c|c|c|c|c|c|}
\hline Items & $\begin{array}{l}\text { Alizad } \\
{[42]}\end{array}$ & $\begin{array}{l}\text { Benninger } \\
{[43]}\end{array}$ & $\begin{array}{l}\text { Capacci } \\
{[44]}\end{array}$ & $\begin{array}{l}\text { Costa-Ribeiro } \\
{[45]}\end{array}$ & $\begin{array}{l}\text { Costa-Ribeiro } \\
\text { [46] }\end{array}$ & $\begin{array}{l}\text { Criminger } \\
{[47]}\end{array}$ & $\begin{array}{l}\text { da Silva } \\
{[48]}\end{array}$ & $\begin{array}{l}\text { Dagan } \\
{[49]}\end{array}$ & $\begin{array}{l}\text { Fernandez- } \\
\text { Lago [50] }\end{array}$ \\
\hline 1. Specific eligibility criteria & 0 & 1 & 0 & 1 & 1 & 1 & 1 & 1 & 1 \\
\hline 2. Subjects random allocation & 1 & 1 & 1 & 1 & 1 & 1 & 1 & 1 & 1 \\
\hline 3. Allocation concealment & 0 & 1 & 1 & 1 & 1 & 0 & 1 & 0 & 0 \\
\hline 4. Similar groups at baseline & 0 & 1 & 0 & 0 & 0 & 0 & 0 & 0 & 0 \\
\hline 5. Blinding of all subjects & 0 & 1 & 0 & 1 & 1 & 1 & 1 & 1 & 0 \\
\hline 6. Blinding of all therapists & 0 & 0 & 0 & 0 & 1 & 0 & 0 & 0 & 0 \\
\hline $\begin{array}{l}\text { 7. Blinding of all assessors } \\
\text { (at least one key outcome) }\end{array}$ & 0 & 1 & 0 & 1 & 1 & 0 & 1 & 1 & 0 \\
\hline $\begin{array}{l}\text { 8. Data measurement from more } \\
\text { than } 85 \% \text { of the subjects initially } \\
\text { allocated to groups } \\
\text { (at least one key outcome) }\end{array}$ & 1 & 1 & 1 & 1 & 1 & 1 & 1 & 1 & 1 \\
\hline $\begin{array}{l}\text { 9. All subjects received the treatment } \\
\text { or control condition as allocated } \\
\text { (at least one key outcome) }\end{array}$ & 1 & 1 & 1 & 1 & 1 & 1 & 1 & 1 & 1 \\
\hline $\begin{array}{l}\text { 10. Between-group comparisons } \\
\text { (at least one key outcome) }\end{array}$ & 0 & 1 & 0 & 1 & 0 & 1 & 1 & 1 & 1 \\
\hline $\begin{array}{l}\text { 11. Point measures and measures of variability } \\
\text { (at least one key outcome) }\end{array}$ & 1 & 1 & 1 & 1 & 1 & 1 & 1 & 1 & 1 \\
\hline Total & 4 & 10 & 5 & 9 & 9 & 7 & 9 & 8 & 6 \\
\hline Items & $\begin{array}{l}\text { Kaski } \\
{[51]}\end{array}$ & $\begin{array}{l}\text { Lattari } \\
{[52]}\end{array}$ & $\begin{array}{l}\text { Mak } \\
{[53]}\end{array}$ & $\begin{array}{l}\text { Manenti } \\
{[54]}\end{array}$ & $\begin{array}{l}\text { Schabrun } \\
\text { [55] }\end{array}$ & $\begin{array}{l}\text { Swank } \\
{[56]}\end{array}$ & $\begin{array}{l}\text { Valentino } \\
{[57]}\end{array}$ & $\begin{array}{l}\text { Verheyden } \\
\text { [58] }\end{array}$ & $\begin{array}{l}\text { Yotnuengnit } \\
\text { [59] }\end{array}$ \\
\hline 1. Specific eligibility criteria & 1 & 1 & 0 & 1 & 1 & 1 & 1 & 1 & 1 \\
\hline 2. Subjects random allocation & 1 & 1 & 1 & 0 & 1 & 1 & 1 & 0 & 1 \\
\hline 3. Allocation concealment & 1 & 1 & 0 & 0 & 1 & 0 & 0 & 0 & 1 \\
\hline 4. Similar groups at baseline & 0 & 1 & 1 & 1 & 1 & 0 & 0 & 0 & 1 \\
\hline 5. Blinding of all subjects & 1 & 1 & 0 & 1 & 1 & 1 & 1 & 1 & 1 \\
\hline 6. Blinding of all therapists & 0 & 0 & 0 & 0 & 0 & 0 & 0 & 0 & 0 \\
\hline $\begin{array}{l}\text { 7. Blinding of all assessors } \\
\text { (at least one key outcome) }\end{array}$ & 1 & 1 & 0 & 1 & 1 & 0 & 1 & 1 & 1 \\
\hline $\begin{array}{l}\text { 8. Data measurement from more than } 85 \% \\
\text { of the subjects initially allocated to groups } \\
\text { (at least one key outcome) }\end{array}$ & 1 & 1 & 1 & 1 & 1 & 1 & 1 & 1 & 1 \\
\hline $\begin{array}{l}\text { 9. All subjects received the treatment } \\
\text { or control condition as allocated } \\
\text { (at least one key outcome) }\end{array}$ & 1 & 1 & 1 & 1 & 1 & 1 & 1 & 1 & 1 \\
\hline $\begin{array}{l}\text { 10. Between-group comparisons } \\
\text { (at least one key outcome) }\end{array}$ & 1 & 1 & 1 & 1 & 0 & 0 & 1 & 1 & 0 \\
\hline $\begin{array}{l}\text { 11. Point measures and measures of variability } \\
\text { (at least one key outcome) }\end{array}$ & 1 & 1 & 0 & 1 & 1 & 1 & 1 & 1 & 1 \\
\hline Total & 9 & 10 & 5 & 8 & 9 & 6 & 8 & 7 & 9 \\
\hline
\end{tabular}

$0.555 ; S E=0.191 ;$ Variance $=0.037 ; 95 \%$ CI $=0.180-0.929$; $\mathrm{Z}=2.902 ; \mathrm{P}=0.004$.

For long-term treatment effects of tDCS, a random effects model meta-analysis on six comparisons failed to show a significant overall effect size $(S M D=0.164 ; S E=$ 0.163 ; Variance $=0.026 ; 95 \% \mathrm{CI}=-0.155-0.483 ; \mathrm{Z}=1.007$; $P=0.314$; Fig. 5). Heterogeneity level for these findings was relatively small: (a) $Q$-statistics $=4.456$ and $P$-value $=$ 0.486 , (b) $T^{2}=0.000$, and (c) $I^{2}=0.00 \%$. In addition, publication bias tests indicated minimal level of risk of bias across included studies: (a) identical overall effect sizes between original and revised funnel plots without any imputed value (Fig. 4b), (b) Egger's regression test: intercept $\beta_{0}=-0.317$ and $P$-value $=0.930$ and (c) Begg and Mazumdar rank correlation test: rank correlation $\tau=$ 0.067 and $P$-value $=0.850$. These findings revealed that tDCS protocols for people with PD did not reveal positive long-term effects on functional locomotion even with minimal variability and risk of bias across six studies.

Further, we conducted two similar sensitivity analyses for long-term effects of tDCS. First sensitivity analysis revealed no significant positive effects from multiple 


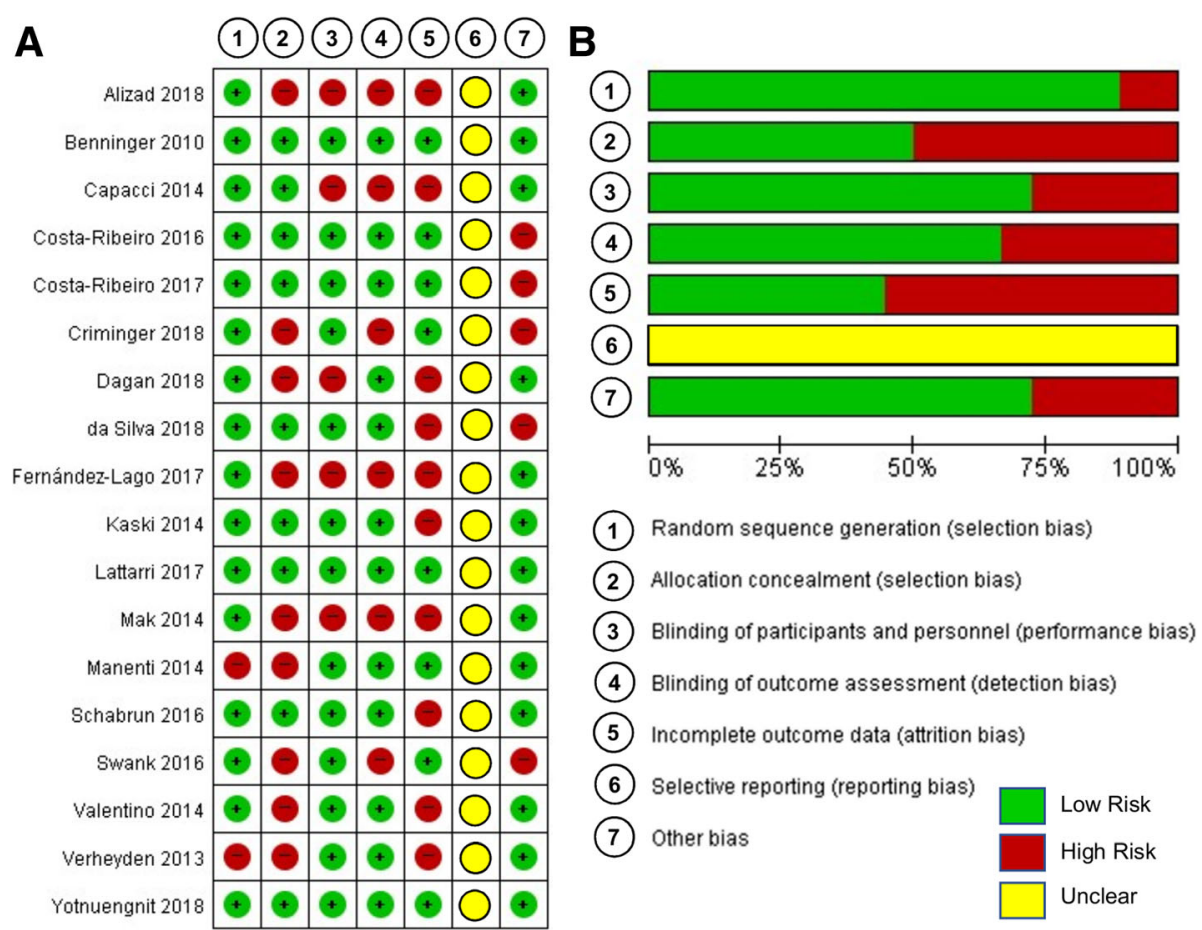

Fig. $\mathbf{2}$ Cochrane risk of bias assessment. a Risk of bias summary and $\mathbf{b}$ Risk of bias graph

comparisons: (a) four gait speed comparisons: $S M D=-0.058 ; \quad S E=0.202 ;$ Variance $=0.041 ; 95 \%$ $\mathrm{CI}=-0.455-0.339 ; \quad \mathrm{Z}=-0.287 ; \quad P=0.774, \quad(\mathrm{~b})$ one TUG time comparison: $S M D=0.353 ; S E=0.430$; Variance $=0.185 ; \quad 95 \% \quad C I=-0.490-1.195 ; \quad Z=0.821$;
$P=0.412$, and (c) one stand walk sit test comparison: $S M D=0.715 ; S E=0.354 ;$ Variance $=0.126 ; 95 \%$ $\mathrm{CI}=0.021-1.410 ; \mathrm{Z}=2.019 ; P=0.044$. Second sensitivity analysis showed no significant positive effects: (a) four difference at posttest comparisons: $S M D=0.175 ; S E=0.223$;

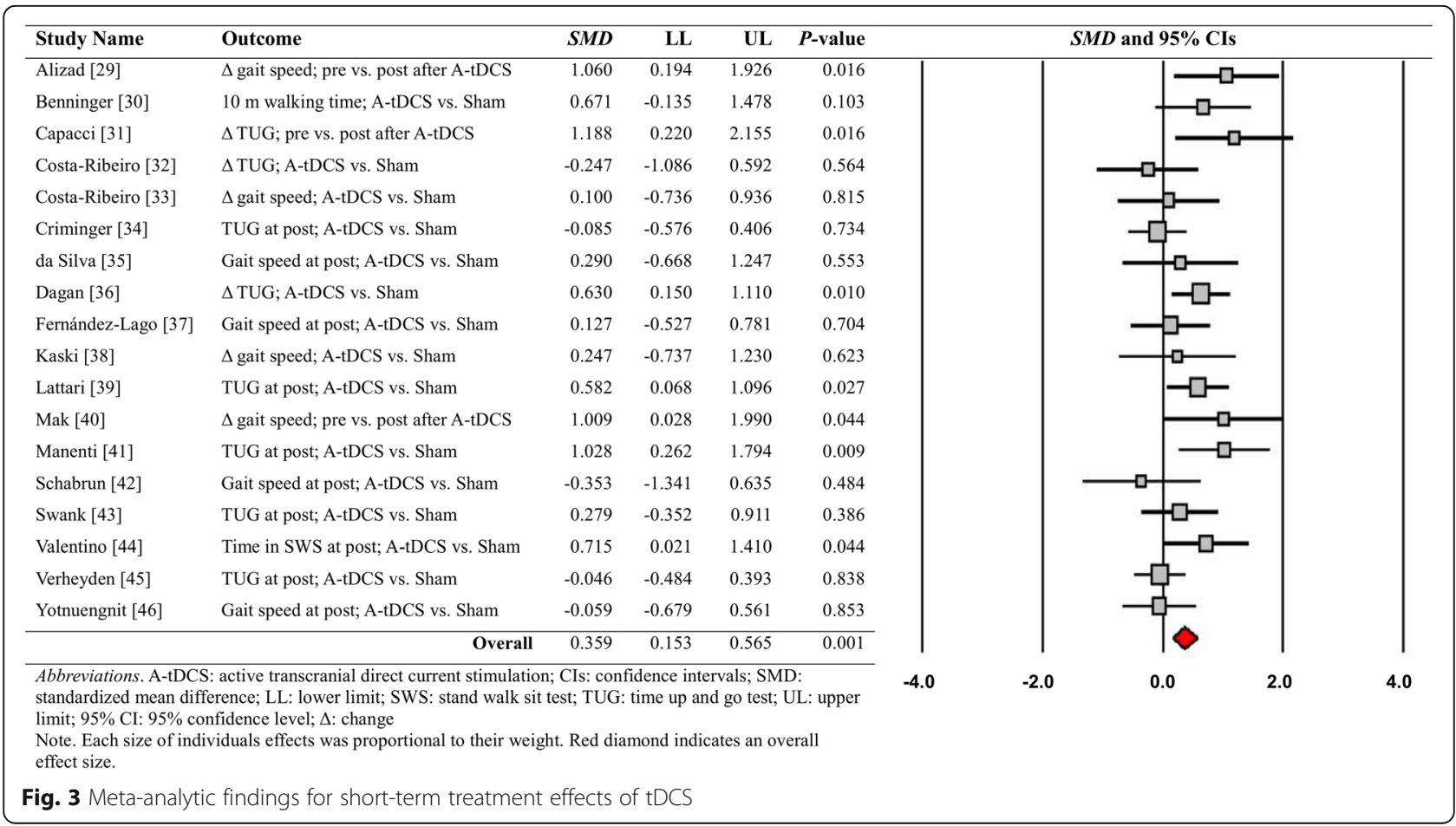



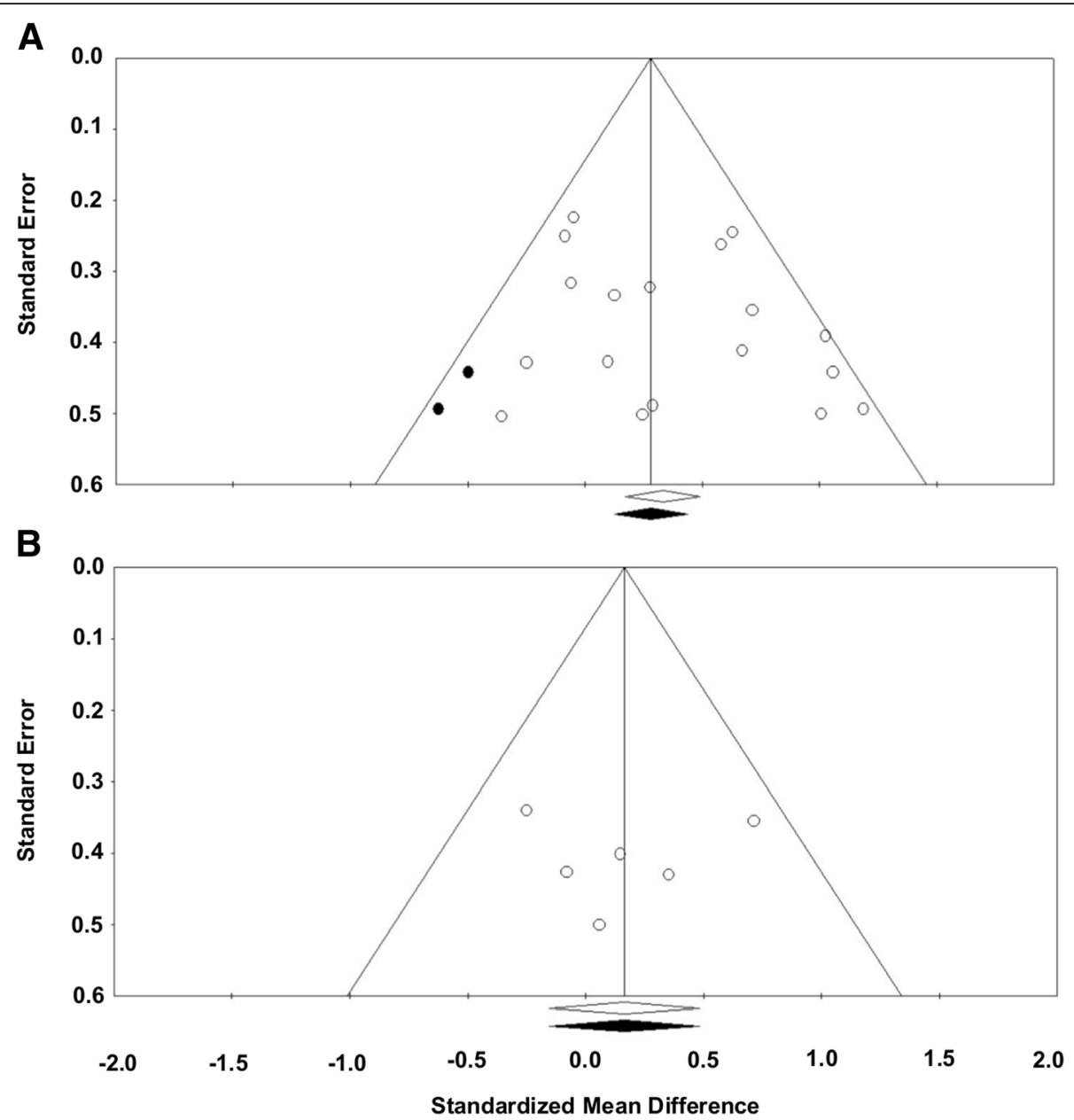

Fig. 4 Publication bias assessments. a Short-term treatment effects of tDCS and $\mathbf{b}$ Long-term treatment effects of tDCS

Variance $=0.050 ; 95 \% \mathrm{CI}=-0.262-0.613 ; \mathrm{Z}=0.786 ; P=$ 0.432 and (b) two changes from baseline to posttest comparisons: $S M D=0.136 ; S E=0.303$; Variance $=0.092 ; 95 \%$ $\mathrm{CI}=-0.458-0.729 ; \mathrm{Z}=0.448 ; P=0.654$.

\section{Moderator variable analysis}

A moderator variable analysis on comparisons for tDCS targeting multiple brain regions versus tDCS targeting a single brain region showed two significant positive overall effect sizes. We performed this moderator variable analysis for 18 short-term effect comparisons. Specific subgroup analyses found: (a) six multiple targeted areas: $S M D=0.527 ; \quad S E=0.194 ; \quad$ Variance $=0.038 ; 95 \% \quad \mathrm{CI}=$ 0.146-0.908; $\mathrm{Z}=2.711 ; P=0.007$ (heterogeneity tests: $Q$ statistics $=9.815$ and $P$-value $=0.081 ; \quad T^{2}=0.11 ; \quad I^{2}=$ $49.06 \%$ ) and (b) 12 single targeted area: $S M D=0.272$; $S E=0.126 ;$ Variance $=0.016 ; 95 \% \mathrm{CI}=0.026-0.518 ; \mathrm{Z}=$ 2.165; $P=0.030$ (heterogeneity tests: $Q$-statistics $=15.324$ and $P$-value $\left.=0.168 ; T^{2}=0.05 ; I^{2}=28.22 \%\right)$. These findings indicate that although both $\mathrm{tDCS}$ protocols revealed significant effect sizes, protocols stimulating multiple brain regions showed relatively more robust treatment effects on functional locomotion than single target tDCS protocols.

\section{Discussion}

The current systematic review and meta-analysis investigated the treatment effects of tDCS interventions on functional locomotion in people with PD. Eighteen total comparisons from the qualified studies showed relatively small positive short-term effects (i.e., immediate posttest $\leq 24 \mathrm{~h}$ after final tDCS interventions) of tDCS, whereas six comparisons revealed no significant long-term effects (i.e., retention periods $\geq 4$ weeks after final tDCS intervention) on functional locomotion in people with PD. Additionally, the moderator variable analysis found that applying tDCS on multiple targeted brain regions (e.g., M1 and PMC; M1 and prefrontal cortex; bilateral M1; bilateral DLPFC) may effectively improve functional locomotion of PD in comparison to tDCS protocols targeting a single brain region. 


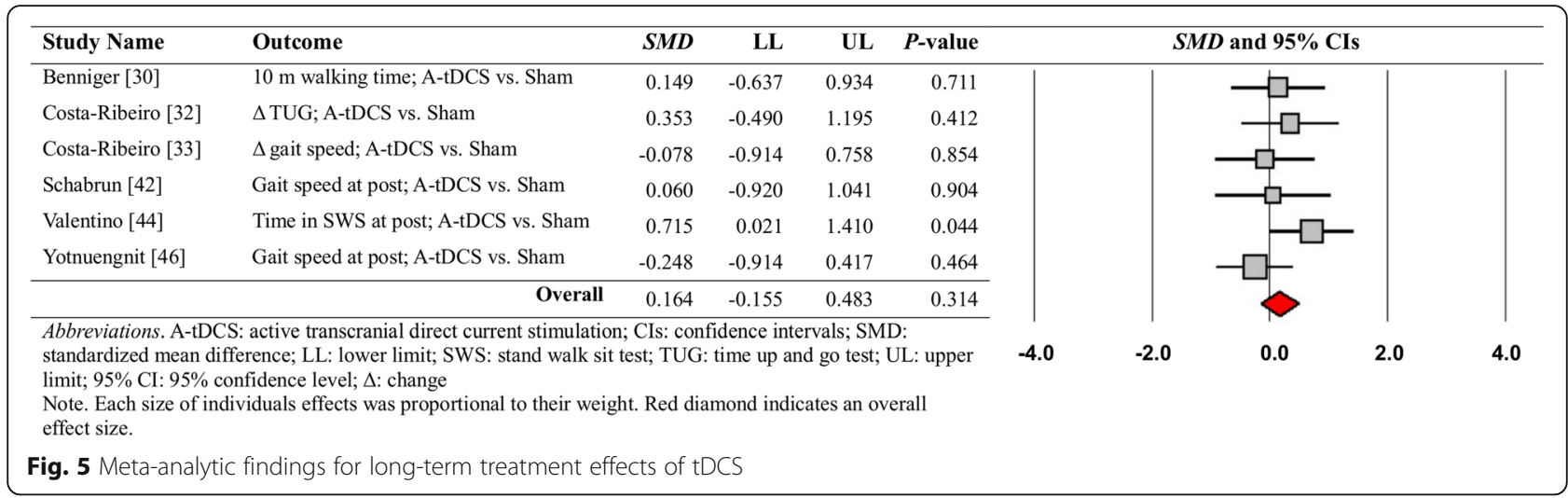

Our meta-analytic findings from 18 qualified studies revealed a significant immediate treatment effect on functional locomotion estimated by temporal gait measurements for 325 people with PD. Although the level of effect size was relatively small [60], these meta-analytic findings with more included studies extended prior findings that tDCS protocols may improve locomotion abilities in people with PD [29-31]. Some tDCS researchers proposed that facilitating cortical excitability using active tDCS may contribute to improvements in motor related symptoms of $\mathrm{PD}[22,30]$. Fregni and colleagues posited that cortical stimulation using tDCS may facilitate the neural connectivity in the cortical and subcortical networks (e.g., the basal gangliathalamocortical motor circuits) presumably improving degenerated functions of the basal ganglia in people with PD [22, 43, 61]. Moreover, the qualified studies in this metaanalysis frequently targeted motor and prefrontal cortices because of crucial role of motor and prefrontal cortical activations in locomotor performance of PD. Specifically, increased M1 activation patterns after anodal tDCS protocols were associated with motor improvements of PD [22]. During normal walking, people with $\mathrm{PD}$ were presumably dependent on cognitive control via increasing DLPFC activations for compensating their impairments in locomotion automaticity. However, given that more challenging walking may require greater DLPFC activation involvement in people with PD, anodal tDCS targeting prefrontal cortices may contribute to successful performance during functional locomotion tasks [23-25]. Finally, similar to previous rTMS studies that reported the release of dopamine in the caudate and putamen for healthy individuals $[26,27]$ and people with PD [28], tDCS interventions triggered on motor and prefrontal cortical regions possibly result in the release of dopamine contributing to functional locomotion improvements.

Our meta-analysis revealed no significant long-term treatment effects from six studies. Previous studies reported that cortical stimulation using tDCS facilitated neural plasticity and long-lasting effects for healthy individuals and people with other neurological disease [62-64]. The current meta-analysis indicated that beneficial effects of tDCS on functional locomotion in people with PD may be primarily manifested in immediate posttests, not in long-term delayed retention tests. These findings were consistent with previous suggestion that anodal tDCS of M1 could positively affect performance adaptation until only $3 \mathrm{~h}$ post training [65]. To elaborate the long-term effects of tDCS protocols on functional locomotion in people with PD, more studies with optimal stimulation protocols for exploring motor learning evidence should be necessary.

Interestingly, the moderator variable analysis revealed that tDCS protocols targeting multiple brain regions may provide better treatment effects on functional locomotion. In our meta-analysis, six studies targeted multiple brain regions such as PFC and M1, PFC, PMC, and M1, and bilateral DLPFC. Given that these motor and prefrontal cortical regions are key brain areas involved in dopaminergic circuits [22, 43,61], stimulating simultaneously these regions presumably activated more neural connectivity and facilitated the release of dopamine in the caudate nucleus contributing to gait improvements $[22,66]$. Specifically, stimulating DLPFC in addition to other motor cortical regions may improve functional locomotion via increasing extra-striatal dopamine release [66] and attenuating functional decoupling between the basal ganglia network and the cognitive control network involving DLPFC [67]. However, establishing specific tDCS protocols for optimizing functional locomotion rehabilitation is still required because of the inconsistent targeted brain regions in the current meta-analytic findings. Comparing treatment effects of a single brain region versus multiple regions targeted by tDCS protocols on PD functional locomotion rehabilitation may be an interesting research focus in the future studies.

Selecting an appropriate hemisphere site for tDCS may be an important issue for optimizing therapeutic effects of tDCS in people with PD. Conventional perspectives suggested that unilateral anodal tDCS targeting M1 of the more affected hemisphere may increase potential treatment effects on motor functions in people with $\mathrm{PD}$ 
$[31,54]$. However, recent studies suggested that bilateral tDCS protocols (i.e., anodal tDCS on the more affected hemispheres and cathodal tDCS on the less affected hemisphere; anodal tDCS on the bilateral hemispheres) are presumably effective for facilitating lower limb functional recovery [43, 68]. Given that various lower limb abilities such as locomotion and postural control were highly associated with controlling the bilateral motor pathway from the affected and unaffected hemispheres [69], bilateral tDCS protocols may improve functional locomotion in people with PD. In this meta-analysis, five studies used anodal tDCS targeting bilateral motor and prefrontal cortical regions and six studies applied anodal tDCS on the central leg areas of M1. Future studies could consider diverse tDCS protocols including dual (motor and prefrontal cortices) and bilateral (more and less affected hemispheres) applications at clinical examination for PD motor recovery.

Despite the positive short-term effects of tDCS on functional locomotion identified in this meta-analysis, these findings are cautiously interpreted because of the possibility of dopaminergic medication suppressing functional locomotion improvements. Fourteen out of 18 total qualified studies in this meta-analysis applied tDCS interventions when people with PD were on medication although four studies did not report medication conditions. Importantly, perhaps the antiparkinson drugs confounded the effects of tDCS because of a ceiling effect [30]. Benninger and colleagues [43] compared tDCS effects on people with PD between "on" and "off" medications, and found greater reduction in bradykinesia during the "off" medication condition. Moreover, a prior study suggested a potential relationship between the required tDCS intensity and the intake of dopaminergic medication: $1 \mathrm{~mA}$ intensity of tDCS with "on" medication improved performance in people with $\mathrm{PD}$ whereas the same tDCS intensity revealed negative effects on gait performance with "off" medication [31]. Taken together, future studies should compare the effects of tDCS between medication conditions to tease apart the interaction effects of dopaminergic medication and tDCS on functional locomotion.

In addition, six out of 18 qualified studies reported freezing of gait (FOG) test results so that participants with severe FOG were excluded from the experiments. However, given that the remaining 12 studies in this meta-analysis did not report FOG conditions of people with PD, the heterogeneous inclusion and exclusion criteria for participants across the included studies may influence functional locomotion outcomes after tDCS protocols. Finally, our sensitivity analyses on the shortterm effects of tDCS revealed comparable effect sizes across three functional locomotion tasks (gait speed vs. TUG time vs. stand walk sit test) and two functional locomotion quantification approaches (difference between active and sham tDCS groups at posttest vs. changes between baseline and posttest after active tDCS as compared with sham stimulation). However, these methodological heterogeneity issues may influence overall treatment effects of tDCS on functional locomotion in people with $\mathrm{PD}$.

\section{Conclusions}

In summary, the current systematic review and metaanalysis provided the evidence that $\mathrm{tDCS}$ interventions reveal short-term intervention benefits for functional locomotion in people with PD. However, the level of effect size was relatively small. Moreover, the treatment effects of active tDCS on functional locomotion of PD may increase when tDCS targeted multiple regions of motor and prefrontal cortices. These findings provide important clinical implications to researchers and clinicians in the utility of tDCS as a potential treatment protocol. To increase our understanding of tDCS treatment effects on functional locomotion, future studies should investigate optimal protocols including ideal targeted brain regions as well as medication conditions for functional locomotion rehabilitation in people with PD.

\section{Additional file}

Additional file 1: PRISMA 2009 Checklist. (DOC 63 kb)

\section{Abbreviations}

Cl: Confidence interval; DBS: Deep brain stimulation; DLPFC: Dorsal lateral prefrontal cortex; M1: Primary motor cortex; NIBS: Non-invasive brain stimulation; PD: Parkinson's disease; PFC: Prefrontal cortex; PMC: Premotor cortex; PRISMA: Preferred Reporting Items for Systematic Reviews and MetaAnalyses; SMA: Supplementary motor area; SMD: Standardized mean difference; tDCS: Transcranial direct current stimulation

\section{Authors' contributions}

$\mathrm{HL}, \mathrm{NK}$, and JHC contributed to statistical analyses, data interpretation, manuscript writing, and revisions. NK, SA, and YS were involved in data collection and manuscript revision. Each author has read and approved the final manuscript.

\section{Funding}

This work was supported by Incheon National University Research Grant in 2018 (2018-0058)

\section{Availability of data and materials}

The datasets generated during the current study are available from the corresponding author on reasonable request.

Ethics approval and consent to participate Not applicable.

Consent for publication

Not applicable.

\section{Competing interests}

The authors declare that they have no competing interests.

\section{Author details}

${ }^{1}$ Division of Sport Science, Neuromechanical Rehabilitation Research Laboratory, Incheon National University, 119 Academy-ro, Yeonsu-gu, 
Incheon, South Korea. ${ }^{2}$ Vector Biomechanics Inc., Yongin, South Korea. ${ }^{3}$ Sport Science Institute, Incheon National University, Incheon, South Korea. ${ }^{4}$ Department of Applied Physiology and Kinesiology, University of Florida, Gainesville, Florida, USA.

\section{Received: 2 February 2019 Accepted: 28 June 2019} Published online: 08 July 2019

\section{References}

1. Berg D, Postuma RB, Bloem B, Chan P, Dubois B, Gasser T, et al. Time to redefine PD? Introductory statement of the MDS task force on the definition of Parkinson's disease. Mov Disord. 2014;29(4):454-62.

2. Jankovic J. Parkinson's disease: clinical features and diagnosis. J Neurol Neurosurg Psychiatry. 2008;79:368-76.

3. Jankovic J, Aguilar LG. Current approaches to the treatment of Parkinson's disease. Neuropsychiatr Dis Treat. 2008;4(4):743-57.

4. Beaulieu-Boire I, Lang AE. Behavioral effects of levodopa. Mov Disord. 2015; 30(1):90-102.

5. Olanow CW, Kieburtz K, Odin P, Espay AJ, Standaert DG, Fernandez HH, et al. Continuous intrajejunal infusion of levodopa-carbidopa intestinal gel for patients with advanced Parkinson's disease: a randomised, controlled, double-blind, double-dummy study. Lancet Neurol. 2014;13(2):141-9.

6. Fasano A, Daniele A, Albanese A. Treatment of motor and non-motor features of Parkinson's disease with deep brain stimulation. Lancet Neurol. 2012;11(5):429-42.

7. Piper M, Abrams GM, Marks WJ Jr. Deep brain stimulation for the treatment of Parkinson's disease: overview and impact on gait and mobility. NeuroRehabilitation. 2005;20(3):223-32.

8. Roper JA, Kang N, Ben J, Cauraugh JH, Okun MS, Hass CJ. Deep brain stimulation improves gait velocity in Parkinson's disease: a systematic review and meta-analysis. J Neurol. 2016;263(6):1195-203.

9. Sydow O. Parkinson's disease: recent development in therapies for advanced disease with a focus on deep brain stimulation (DBS) and duodenal levodopa infusion. FEBS J. 2008;275(7):1370-6.

10. Weaver FM, Follett K, Stern M, Hur K, Harris C, Marks WJ, et al. Bilateral deep brain stimulation vs best medical therapy for patients with advanced Parkinson disease: a randomized controlled trial. JAMA. 2009;301(1):63-73.

11. Koch $\mathrm{G}$. Do studies on cortical plasticity provide a rationale for using noninvasive brain stimulation as a treatment for Parkinson's disease patients? Front Neurol. 2013;4:180.

12. Tahtis $V$, Kaski D. Parkinson's disease treatments: focus on transcranial direct current stimulation (tDCS). J Parkinsonism Restl. 2017;7:55-70.

13. Nitsche MA, Paulus W. Excitability changes induced in the human motor cortex by weak transcranial direct current stimulation. J Physiol. 2000;527(Pt 3):633-9.

14. Hordacre B, Moezzi B, Ridding MC. Neuroplasticity and network connectivity of the motor cortex following stroke: a transcranial direct current stimulation study. Hum Brain Mapp. 2018;39(8):3326-39.

15. Monte-Silva K, Kuo MF, Hessenthaler S, Fresnoza S, Liebetanz D, Paulus W, et al. Induction of late LTP-like plasticity in the human motor cortex by repeated non-invasive brain stimulation. Brain Stimul. 2013;6(3):424-32.

16. Polania R, Nitsche MA, Paulus W. Modulating functional connectivity patterns and topological functional organization of the human brain with transcranial direct current stimulation. Hum Brain Mapp. 2011;32(8):1236-49.

17. Quartarone A, Morgante F, Bagnato S, Rizzo V, Sant'Angelo A, Aiello E, et al. Long lasting effects of transcranial direct current stimulation on motor imagery. Neuroreport. 2004;15(8):1287-91.

18. Lu C, Wei Y, Hu R, Wang Y, Li K, Li X. Transcranial direct current stimulation ameliorates behavioral deficits and reduces oxidative stress in 1-Methyl-4Phenyl-1,2,3,6-Tetrahydropyridine-induced mouse model of Parkinson's disease. Neuromodulation. 2015;18(6):442-6 discussion 447

19. Li H, Lei $X$, Yan T, Li H, Huang B, Li L, et al. The temporary and accumulated effects of transcranial direct current stimulation for the treatment of advanced Parkinson's disease monkeys. Sci Rep. 2015:5:12178.

20. Tanaka T, Takano Y, Tanaka S, Hironaka N, Kobayashi K, Hanakawa T, et al. Transcranial direct-current stimulation increases extracellular dopamine levels in the rat striatum. Front Syst Neurosci. 2013;7:6.

21. Doruk D, Gray Z, Bravo GL, Pascual-Leone A, Fregni F. Effects of tDCS on executive function in Parkinson's disease. Neurosci Lett. 2014:582:27-31.

22. Fregni F, Boggio PS, Santos MC, Lima M, Vieira AL, Rigonatti SP, et al. Noninvasive cortical stimulation with transcranial direct current stimulation in Parkinson's disease. Mov Disord. 2006;21(10):1693-702.
23. Maidan I, Nieuwhof F, Bernad-Elazari H, Reelick MF, Bloem BR, Giladi N, et al. The role of the frontal lobe in complex walking among patients with Parkinson's disease and healthy older adults: an fNIRS study. Neurorehabil Neural Repair. 2016;30(10):963-71.

24. Stuart S, Vitorio R, Morris R, Martini DN, Fino PC, Mancini M. Cortical activity during walking and balance tasks in older adults and in people with Parkinson's disease: a structured review. Maturitas. 2018;113:53-72.

25. Vitorio R, Stuart $S$, Rochester $L$, Alcock L, Pantall A. fNIRS response during walking - Artefact or cortical activity? A systematic review. Neurosci Biobehav. 2017:83:160-72.

26. Strafella AP, Paus T, Barrett J, Dagher A. Repetitive transcranial magnetic stimulation of the human prefrontal cortex induces dopamine release in the caudate nucleus. J Neurosci. 2001:21(15):RC157.

27. Strafella AP, Paus T, Fraraccio M, Dagher A. Striatal dopamine release induced by repetitive transcranial magnetic stimulation of the human motor cortex. Brain. 2003;126(Pt 12):2609-15.

28. Strafella AP, Ko JH, Grant J, Fraraccio M, Monchi O. Corticostriatal functional interactions in Parkinson's disease: a rTMS/[11C] raclopride PET study. Eur J Neurosci. 2005:22(11):2946-52.

29. Goodwill AM, Lum JAG, Hendy AM, Muthalib M, Johnson L, Albein-Urios N, et al. Using non-invasive transcranial stimulation to improve motor and cognitive function in Parkinson's disease: a systematic review and metaanalysis. Sci Rep. 2017;7(1):14840.

30. Fregni F, Simon DK, Wu A, Pascual-Leone A. Non-invasive brain stimulation for Parkinson's disease: a systematic review and meta-analysis of the literature. J Neurol Neurosurg Psychiatry. 2005;76(12):1614-23.

31. Broeder S, Nackaerts E, Heremans E, Vervoort G, Meesen R, Verheyden G, et al. Transcranial direct current stimulation in Parkinson's disease: neurophysiological mechanisms and behavioral effects. Neurosci Biobehav Rev. 2015;57:105-17.

32. Liberati A, Altman DG, Tetzlaff J, Mulrow C, Gotzsche PC, loannidis JP, et al. The PRISMA statement for reporting systematic reviews and meta-analyses of studies that evaluate health care interventions: explanation and elaboration. PLoS Med. 2009;6(7):e1000100.

33. Maher CG, Sherrington C, Herbert RD, Moseley AM, Elkins M. Reliability of the PEDro scale for rating quality of randomized controlled trials. Phys Ther. 2003:83(8):713-21.

34. Lundh A, Gotzsche PC. Recommendations by Cochrane review groups for assessment of the risk of bias in studies. BMC Med Res Methodol. 2008;8:22.

35. Borenstein M, Hedges LV, Higgins JP, Rothstein HR. Introduction to metaanalysis. New York: Wiley; 2009.

36. Spiegelhalter DJ, Abrams KR, Myles JP. Bayesian approaches to clinical trials and health-care evaluation. Chichester: Wiley; 2004.

37. Ren S, Oakley JE, Stevens JW. Incorporating genuine prior information about between-study heterogeneity in random effects pairwise and network meta-analyses. Med Decis Mak. 2018;38(4):531-42.

38. Higgins JP, Thompson SG. Quantifying heterogeneity in a meta-analysis. Stat Med. 2002;21(11):1539-58

39. Duval S, Tweedie R. Trim and fill: a simple funnel-plot-based method of testing and adjusting for publication bias in meta-analysis. Biometrics. 2000;56(2):455-63.

40. Egger M, Davey Smith G, Schneider M, Minder C. Bias in meta-analysis detected by a simple, graphical test. BMJ. 1997;315(7109):629-34.

41. Begg CB, Mazumdar M. Operating characteristics of a rank correlation test for publication bias. Biometrics. 1994;50(4):1088-101.

42. Alizad V, Meinzer M, Frossard L, Polman R, Smith S, Kerr G. Gait speed after applying anodal-transcranial direct current stimulation in people with Parkinson's disease? Preliminary results. Mov Disord. 2018;33:S102.

43. Benninger DH, Lomarev M, Lopez G, Wassermann EM, Li X, Considine E, et al. Transcranial direct current stimulation for the treatment of Parkinson's disease. J Neurol Neurosurg Psychiatry. 2010;81(10):1105-11.

44. Capecci M, Andrenelli E, Orni C, Ceravolo MG. Bilateral prefrontal transcrania direct current stimulation (tDCS) in Parkinson's disease: a placebo controlled trial. Mov Disord. 2014:29:S229-S30.

45. Costa-Ribeiro A, Maux A, Bosford T, Tenorio Y, Marques D, Carneiro M, et al. Dopamine-independent effects of combining transcranial direct current stimulation with cued gait training on cortical excitability and functional mobility in Parkinson's disease. J Rehabil Med. 2016:48(9):819-23.

46. Costa-Ribeiro A, Maux A, Bosford T, Aoki Y, Castro R, Baltar A, et al. Transcranial direct current stimulation associated with gait training in Parkinson's disease: a pilot randomized clinical trial. Dev Neurorehabil. 2017;20(3):121-8.

47. Criminger C, Swank C, Almutairi S, Mehta J. Transcranial direct current stimulation plus concurrent activity may influence task prioritization during 
walking in people with Parkinson's disease - initial findings. J Parkinsonism Restl. 2018;8:25-32.

48. da Silva DCL, L T, SFA D, Horsczaruk CHR, Pedron CA, Rodrigues EDC, et al. Effects of acute transcranial direct current stimulation on gait kinematics of individuals with Parkinson disease. Top Geriatr Rehabil. 2018:34:262-8.

49. Dagan M, Herman T, Harrison R, Zhou J, Giladi N, Ruffini G, et al. Multitarget transcranial direct current stimulation for freezing of gait in Parkinson's disease. Mov Disord. 2018;33(4):642-6.

50. Fernandez-Lago H, Bello O, Mora-Cerda F, Montero-Camara J, Fernandez-del-Olmo MA. Treadmill walking combined with anodal Transcranial direct current stimulation in Parkinson disease: a pilot study of kinematic and neurophysiological effects. Am J Phys Med Rehabil. 2017;96(11):801-8.

51. Kaski D, Dominguez RO, Allum JH, Islam AF, Bronstein AM. Combining physical training with transcranial direct current stimulation to improve gait in Parkinson's disease: a pilot randomized controlled study. Clin Rehabil. 2014;28(11):1115-24.

52. Lattari E, Costa SS, Campos C, de Oliveira AJ, Machado S, Neto GAM Can transcranial direct current stimulation on the dorsolateral prefrontal cortex improves balance and functional mobility in Parkinson's disease? Neurosci Lett. 2017;636:165-9.

53. Mak M, Yu L. Effects of transcranial direct current stimulation on dual-task gait performance in patients with Parkinson's disease. Clin Neurophysiol. 2014;125:S127.

54. Manenti R, Brambilla M, Rosini S, Orizio I, Ferrari C, Borroni B, et al. Time up and go task performance improves after transcranial direct current stimulation in patient affected by Parkinson's disease. Neurosci Lett. 2014;580:74-7.

55. Schabrun SM, Lamont RM, Brauer SG. Transcranial direct current stimulation to enhance dual-task gait training in Parkinson's disease: a pilot RCT. PLoS One. 2016;11(6):e0158497.

56. Swank C, Mehta J, Criminger C. Transcranial direct current stimulation lessens dual task cost in people with Parkinson's disease. Neurosci Lett. 2016;626:1-5.

57. Valentino F, Cosentino G, Brighina F, Pozzi NG, Sandrini G, Fierro B, et al. Transcranial direct current stimulation for treatment of freezing of gait: a cross-over study. Mov Disord. 2014;29(8):1064-9.

58. Verheyden G, Purdey J, Burnett M, Cole J, Ashburn A. Immediate effect of transcranial direct current stimulation on postural stability and functional mobility in Parkinson's disease. Mov Disord. 2013; 28(14):2040-1.

59. Yotnuengnit P, Bhidayasiri R, Donkhan R, Chaluaysrimuang J, Piravej K. Effects of Transcranial direct current stimulation plus physical therapy on gait in patients with Parkinson disease: a randomized controlled trial. Am J Phys Med Rehabil. 2018:97(1):7-15.

60. Cohen J. A power primer. Psychol Bull. 1992;112(1):155-9.

61. Ferrucci R, Mameli F, Ruggiero F, Priori A. Transcranial direct current stimulation as treatment for Parkinson's disease and other movement disorders. Basal Ganglia. 2016;6:53-61.

62. Cuypers K, Leenus DJF, den Berg FE, Nitsche MA, Thijs H, Wenderoth N, et al. Is motor learning mediated by tDCS intensity? PLoS One. 2013;8(6):e67344.

63. Kang N, Summers JJ, Cauraugh JH. Transcranial direct current stimulation facilitates motor learning post-stroke: a systematic review and meta-analysis. J Neurol Neurosurg Psychiatry. 2016;87(4):345-55.

64. Boggio PS, Ferrucci R, Mameli F, Martins D, Martins O, Vergari M, et al. Prolonged visual memory enhancement after direct current stimulation in Alzheimer's disease. Brain Stimul. 2012;5(3):223-30.

65. Reis J, Schambra HM, Cohen LG, Buch ER, Fritsch B, Zarahn E, et al. Noninvasive cortical stimulation enhances motor skill acquisition over multiple days through an effect on consolidation. Proc Natl Acad Sci U S A. 2009;106(5):1590-5.

66. Cho SS, Strafella AP. rTMS of the left dorsolateral prefrontal cortex modulates dopamine release in the ipsilateral anterior cingulate cortex and orbitofrontal cortex. PLoS One. 2009;4(8):e6725.

67. Shine JM, Matar E, Ward PB, Frank MJ, Moustafa AA, Pearson M, et al. Freezing of gait in Parkinson's disease is associated with functional decoupling between the cognitive control network and the basal ganglia. Brain. 2013;136(Pt 12:3671-81.
68. Hadoush H, Al-Jarrah M, Khalil H, Al-Sharman A, Al-Ghazawi S. Bilateral anodal transcranial direct current stimulation effect on balance and fearing of fall in patient with Parkinson's disease. NeuroRehabilitation. 2018;42(1):63-8.

69. Luft AR, Smith GV, Forrester L, Whitall J, Macko RF, Hauser TK, et al. Comparing brain activation associated with isolated upper and lower limb movement across corresponding joints. Hum Brain Mapp. 2002;17(2):131-40.

\section{Publisher's Note}

Springer Nature remains neutral with regard to jurisdictional claims in published maps and institutional affiliations.

\section{Ready to submit your research? Choose BMC and benefit from:}

- fast, convenient online submission

- thorough peer review by experienced researchers in your field

- rapid publication on acceptance

- support for research data, including large and complex data types

- gold Open Access which fosters wider collaboration and increased citations

- maximum visibility for your research: over $100 \mathrm{M}$ website views per year

At $\mathrm{BMC}$, research is always in progress.

Learn more biomedcentral.com/submissions 